\title{
GRILLE DECOMPOSITION AND CONVEXIFICATION THEOREMS FOR COMPACT METRIC LOCALLY CONNECTED CONTINUA
}

\author{
EDWIN E. MOISE
}

A convex metric space is a metric space in which for each two points $a$ and $b$ there is a point $c$, different from $a$ and $b$, such that $d(a, b)$ $=d(a, c)+d(c, b)$, where $d$ is the distance function. Menger has raised the question whether for every Peano space (that is, compact, metric, locally connected continuum) it is possible to define a distance function (preserving the original topology) with respect to which the space is convex. ${ }^{1}$ In the present paper, this question is answered in the affirmative. For a general discussion of the problem and various partial solutions of it, see Menger ${ }^{2}$ and Blumenthal. ${ }^{3}$ After Blumenthal's book was written, Beer ${ }^{4}$ and Bing ${ }^{5}$ established convexification theorems for the one-dimensional and finite-dimensional cases respectively.

Only a short time after the present paper was written, Bing independently obtained a proof of the convexification theorem. His paper will be published in this Bulletin.

As Menger has shown ${ }^{6}$ every compact convex metric space is locally connected. An affirmative answer to Menger's question therefore shows that for compact metric spaces, local connectedness and the existence of a convex metric are equivalent.

Theorems 1 and 2, our principal preliminary results, may be of interest independently of the use made of them here; they do not appear to be readily deducible from the convexification theorem, and their conclusions are considerably stronger than our present purposes require.

Theorem 1. Let $S$ be a Peano space and let $R, R^{\prime}$, and $D$ be open sets such that (1) $R \subset R^{\prime} \subset D$, (2) the closures of $\beta(R)-\beta(D)$ and

Presented to the Society, September 10,1948; received by the editors November 26, 1948.

${ }^{1} \mathrm{~K}$. Menger, Untersuchungen über allgemeine Metrik, Math. Ann. vol. 100 (1928) pp. 81 and 98.

${ }^{2}$ Menger, loc. cit. p. $96 \mathrm{ff}$.

${ }^{3}$ Leonard M. Blumenthal, Distance geometries, University of Missouri Studies, vol. 13, 1938, No. 2, p. 43.

${ }^{4} \mathrm{G}$. Beer, Beweis des Satzes, dass jede im kleinen zusammenhängende Kurve konvex metrisiert werden kann, Fund. Math. vol. 31 (1938) p. 281.

${ }^{5} \mathrm{R}$. Bing, A convex metric for a locally connected continuum, Bull. Amer. Math. Soc. vol. 55 (1949) pp. 812-819.

${ }^{6}$ Menger, loc. cit., p. 98. 
$\beta\left(R^{\prime}\right)-\beta(D)$ do not intersect, and (3) $D$ is locally connected at each point of $\beta(D) .^{7}$ Then there is an open set $R^{\prime \prime}$ such that (1) $R \subset R^{\prime \prime} \subset R^{\prime}$, and (2) $R^{\prime \prime}$ and $D-\bar{R}^{\prime \prime}$ are locally connected at each point of $\beta\left(R^{\prime \prime}\right) .^{8}$

Proof. The proof of this theorem will involve some rather complicated definitions; and it may be well to explain their motivation by giving a very rough sketch of the proof. We wish to obtain a sequence $U_{1}, U_{2}, \cdots$ of open sets lying between $R$ and $R^{\prime}$ (in the sense that $\left.R \subset U_{i} \subset R^{\prime}\right)$ such that the sequence $U_{1}, U_{3}, \cdots$ is monotone ascending and $U_{2}, U_{4}, \cdots$ is monotone descending, and a sequence $G_{1}, G_{2}, \cdots$ of finite collections of open sets, each a refinement of the one before, with meshes ${ }^{9}$ approaching 0 , covering the annuli between successive terms of the $U$-sequence. It will turn out that $R^{\prime \prime}$ is the sum of the sets $U_{2 i+1}$ and that the closure of $\beta\left(R^{\prime \prime}\right)-\beta(D)$ is the common part of the sets $G_{i}^{*}{ }^{10} \mathrm{We}$ shall want to use the elements of the collections $G_{i}$ to establish local connectivity for $R^{\prime \prime}$ on $\beta\left(R^{\prime \prime}\right)$. We therefore want the sets $g \cdot U_{2 i+1}$ (where $g$ belongs to a term of the $G$-sequence) to be in some sense "as nearly connected as possible." We are forced to give rather complicated criteria of such "optimum connectness." Furthermore, at each stage we wish to define an element of the $U$-sequence so as to give ourselves a manageable situation for the next stage. Definition $F$ gives a precise formulation of this condition. Finally, in Lemmas 4 and 5, we show that by doing our best at each stage, we do well enough.

Definition A. By $U: V$ we mean that $U$ and $V$ satisfy the hypothesis of the theorem for $R$ and $R^{\prime}$.

Definition B. If $U$ is an open set lying in $D$, then by $B(U)$ we mean the closure of $\beta(U)-\beta(D)$.

Definition $C$. If $B$ is a closed set in $\bar{D}$, then an admissible covering

${ }^{7}$ If $A$ is a set, then $\beta(A)$ is the boundary of $A$. If $A$ and $B$ are sets, then $A-B$ denotes $A-A \cdot B$ whether $B$ is a subset of $A$ or not. A set $M$ is locally connected at a point $p$ (which may or may not belong to $M$ ) if for each open set $U$ containing $p$, there is an open $U^{\prime}$ containing $p$ and lying in $U$ such that $U^{\prime} \cdot M$ is connected. (See R. L. Wilder, Topology of manifolds, Amer. Math. Soc. Colloquium Publications, vol. 32.) An open subset of a compact space is uniformly locally connected if and only if it is locally connected at every point of its closure; the notion of local connectivity used in Theorem 1 therefore is equivalent to the more cumbersome notion of uniform local connectedness.

${ }^{8}$ This is a strengthened form of a theorem of R. L. Wilder, loc. cit. Theorem III 3.5; Wilder's theorem states that if $R$ and $R^{\prime}$ are open subsets of a Peano space and $\bar{R} \subset R^{\prime}$, then there is a uniformly locally connected open set $R^{\prime \prime}$ lying between $R$ and $R^{\prime}$.

- The mesh of a collection $G$ of sets is the least upper bound of the diameters of the elements of $G$.

${ }^{10}$ If $G$ is a collection of sets, then $G^{*}$ is the set of all elements of elements of $G$. 
of $B$ is a finite collection $G$ of open sets covering $B$ such that for each $g$ of $G, g \cdot D$ is connected.

Definition D. Suppose that $U: V$ and that (1) $G$ is an admissible covering of $B(U)+B(V)$ such that (2) $D C U+(S-\bar{V})+G^{*}$. Then $G$ is contractible with respect to $U$ and $V$ if there is an admissible covering $T(G)$ (where $T$ is one-to-one) having properties (1) and (2) such that if $g^{\prime}=T(g) \in T(G)$, then $\bar{g}^{\prime} \subset g .{ }^{11} T(G)$ is called a contraction of $G$. If we require merely that $g^{\prime} \subset g$, then $T(G)$ is a weak contraction of $G$.

LeMma 1. If $U: V$ and $G_{I}$ and $G_{0}$ satisfy conditions (1) and (2) of Definition $\mathrm{D}$, and $G_{I}=T\left(G_{0}\right)$, then there is $a G^{\prime}$ which is a contraction of $G_{0}$ such that $G_{I}$ is a contraction of $G^{\prime}$.

Definition E. If $U: V($ or $V: U), G$ is contractible with respect to $U$ and $V, G^{\prime}$ is an admissible covering of $B(U)$, and $G^{\prime} \gg G,{ }^{12}$ then a jumpless extension of $V$ with respect to $U, G$, and $G^{\prime}$ is an open set $V^{\prime}$ such that (1) $U: V^{\prime}: V$ (or $V: V^{\prime}: U$ ), (2) for each point $p$ of $G^{*} \cdot\left(D-\bar{V}^{\prime}\right)$ (or of $\left.G^{*} \cdot V^{\prime}\right)$ there is a $g$ of $G$ such that some component of $g \cdot\left(D-\bar{V}^{\prime}\right)$ (or of $\left.g \cdot V^{\prime}\right)$ contains $p$ and has a limit point in $B(V)$, and (3) $G^{\prime}$ is contractible with respect to $U$ and $V^{\prime}$.

Definition F. Let $U: V$ (or $V: U$ ) and let $\Gamma$ be contractible with respect to $U$ and $V$. Let $\epsilon$ be a positive number. Then $U$ is said to be $\epsilon$-approachable from $V$ through $\Gamma$ if there is an open set $V^{\prime}$ and a collection $G$ which is contractible with respect to $U$ and $V^{\prime}$ such that (1) $U: V^{\prime}: V$, (or $V: V^{\prime}: U$ ), (2) $G \gg \Gamma$, (3) $G$ has mesh less than $\epsilon$, and (4) $V^{\prime}$ is a jumpless extension of $V$ with respect to $U, \Gamma$, and $G$. Under these conditions, $V^{\prime}$ is an e-approach to $U$ through $\Gamma$.

LeMma 2. Let $U: V$, let $G_{I}$ and $G_{0}$ be contractible with respect to $U$ and $V$ such that $G_{I}$ is a contraction of $G_{0}$, and let $\epsilon$ be a positive number. Then there is a set $U^{\prime}$ and a collection $\Gamma$ which is contractible with respect to $U$ and $V$ such that (1) $\Gamma$ is a contraction of $G_{0},(2) G_{I}$ is a weak contraction of $\Gamma$, (3) if $\gamma$ and $g$ are corresponding elements of $\Gamma$ and $G_{I}$ under the appropriate weak contraction, then no point of $B(U)$ is a limit point of $\gamma-g$, (4) $U^{\prime}$ is a jumpless extension of $U$ with respect to $V, \Gamma$, and $\Gamma,{ }^{13}$ and (5) $U^{\prime}$ is $\epsilon$-approachable from $V$ through $\Gamma$.

Proof of Lemma. Let $G^{\prime}$ be an admissible covering of $B(U)$ and

${ }^{11}$ For reasons of typographical convenience, the superior bar, indicating closure, has not been placed over primes or subscripts. For example, $\bar{g}^{\prime}, \bar{G}^{*}$, and $\bar{U}_{1}$ are the elesures of $g^{\prime}, G^{*}$, and $U_{1}$.

${ }_{12} G^{\prime} \gg G$ means that $G^{\prime}$ is a closure refinement of $G$; that is, the closure of each element of $G^{\prime}$ lies in some element of $G$.

${ }^{13}$ Here $\Gamma$ plays the parts of both the $G$ and the $G^{\prime}$ of Definition $E$. 
let $W$ be an open set such that (1) $U: W: V$, (2) $G^{\prime}$ is contractible with respect to $U$ and $W,(3) \bar{G}^{\prime *} \cdot B(V)$ is empty, (4) $G^{\prime} \gg G_{I}$, and (5) the mesh of $G^{\prime}$ is less than $\epsilon$. Let $Z$ be the interior of the set of all points $p$ of $D-\bar{U}$ for which there does not exist a $g$ of $G_{I}$ such that some component of $g \cdot(D-\bar{U})$ contains $p$ and has a limit point in $B(V) . Z$ is clearly open, and no point of $B(V)$ is a limit point of $U^{\prime \prime}=W+Z$. Let $G^{\prime \prime}$ be an admissible covering of $B\left(U^{\prime \prime}\right)$ and a closure refinement of $G_{I}$, of mesh such that $\bar{G}^{\prime \prime *} \cdot B(V)$ is empty. Let $G$ be $G^{\prime}+G^{\prime \prime}$.

It is now true that there is an open set $U^{\prime}$, and a collection $\Gamma$ such that $U^{\prime}, \Gamma$, and $G$ satisfy the conclusion of the lemma. Let $X$ be an open set such that $U^{\prime \prime}: X: V$ and $G$ is contractible with respect to $U^{\prime \prime}$ and $X$. Let $\Gamma$ be a collection satisfying (1), (2), and (3) of the conclusion of the lemma, and having the further property that for each $\gamma$ of $\Gamma$ all but a finite number of components of $g \cdot(D-\bar{X})$ either lie in $G^{*}$ or have a limit-point in $B(V)$. (Note that to obtain each $\gamma$ of $\Gamma$ from the corresponding $g$ of $G_{I}$, we need only add to $g$ the sum of a finite number of sufficiently small neighborhoods of points of the boundary of $G^{*}$.) If one of these exceptional components can be connected to $B(V)$ in $g \cdot(D-\bar{X})$ merely by making the set $X$ smaller, we do so. The hypothesis for $Z$ shows that by repeating this process a finite number of times, we obtain the desired $U^{\prime}$.

Definition G. If $U$ and $g$ are sets, then $N(U, g)$ is the number of components of $U \cdot g$. If $U$ is a set and $G$ is a collection of sets, then $I(U, G)$ is $\sum_{o \in G} N(U, g)$.

LEMma 3. If $U: U_{I}: V_{0}: V$ and $G_{0}$ and $G_{I}=T\left(G_{0}\right)$ are contractible with respect to $U$ and $V$, then there are sets $U^{\prime}$ and $V^{\prime}$, and a collection $G$ which is contractible with respect to $U$ and $V$ such that (1) $U: U^{\prime}$ $: U_{I}: V_{0}: V^{\prime}: V$, (2) $G_{I}$ and $G$ are contractions of $G$ and $G_{0}$ respectively, and (3) $I\left(U^{\prime}, G\right)$ and $I\left(D-\bar{V}^{\prime}, G\right)$ are both finite.

Proof of Lemma. Let $g$ belong to $G_{I}$. Since $g \cdot D$ is connected, each component of $U \cdot g$ has a limit point in $\bar{g} \cdot B(U)$. Let $H_{\theta}$ be a finite collection of open sets such that (1) for each $h$ of $H_{\theta}, h \cdot D$ is connected, (2) if $h \in H_{g}$ and $g^{\prime} \in G_{I}$, then $B(h)$ intersects $B\left(g^{\prime}\right)$ only if $h$ intersects $B\left(g^{\prime}\right)$, (3) $\bar{h} \cdot B\left(U_{I}\right)$ is empty, (4) $H_{g}$ covers $g \cdot B(U)$, (5) if $g^{\prime}$ and $g^{\prime \prime}$ belong to $G_{I}$ and $G_{0}$ respectively and $\bar{g}^{\prime}$ lies in $g^{\prime \prime}$, then no element of $H_{g}$ contains a point of $B\left(G^{\prime}\right)$ and a point of $B\left(g^{\prime \prime}\right)$, and (6) there is a collection $Z\left(H_{g}\right)$ having properties (1), (2), (3), (4), and (5) such that for each $h$ of $H_{g}, \bar{h}$ lies in $Z(h)$. (Here $Z$ denotes a transformation throwing $H_{g}$ onto $Z\left(H_{g}\right)$.)

Now let $U_{g}$ be the sum of $U$ and the elements $g$ of $H_{g}$ which intersect $g \cdot U$. Let $Y(g)$ be $g+U_{g}$. For each $g^{\prime}$ of $G_{I}-g$, let $Y\left(g^{\prime}\right)$ be the 
sum of $g^{\prime}$ and the elements of $H_{g}$ that intersect $U \cdot g^{\prime}$. It is now true that $N\left(U_{g}, Y(g)\right)$ is finite and $I\left(U_{g}, Y\left(G_{I}-g\right)\right) \leqq I\left(U, G_{I}-g\right)$. Moreover, there is a collection $Y^{\prime}\left(G_{I}\right)$, of which $Y\left(G_{I}\right)$ is a contraction, which has all the same properties, such that $Y^{\prime}\left(G_{I}\right)$ is a contraction of $G_{I}$ and $G_{0}$ is a contraction of $Y^{\prime}\left(G_{I}\right)$, with respect to $U$ and $V$, and also with respect to $U_{g}$ and $V$.

Clearly, by a finite number of such operations, first for $U$ and then for $V$, we obtain the sets $U^{\prime}, V^{\prime}$ and the collection $G$ required in the conclusion of the lemma.

Definition $H$. Let $U_{1}: U_{2}$ and let $G$ be contractible with respect to $U_{1}$ and $U_{2}$. Then for each $g$ of $G$, the index Ind $\left(U_{2}, U_{1}, g\right)$ of $U_{2}$ with respect to $U_{1}$ and $g$ is the number of components of $g \cdot U_{2}$ that have a limit point in $B\left(U_{1}\right)$; and the index Ind $\left(U_{2}, U_{1}, G\right)$ of $U_{2}$ with respect to $U_{1}$ and $G$ is $\sum_{g \in G}$ Ind $\left(U_{2}, U_{1}, g\right)$. (Obviously Ind $\left(U_{2}, U_{1}, G\right)$ $\leqq I\left(U_{2}, G\right)$.) An analogous definition of Ind $\left(U_{2}, U_{1}, G\right)$ may be given for the case $U_{2}: U_{1}{ }^{14}$

With the help of the above lemmas, we may now define a sequence $U_{1}, U_{2}, \cdots$ of open sets and a triangular sequence $G_{i, j}$ of finite collection of open sets $(i=1,2, \cdots ; j=i, i+1, \cdots)$ satisfying the following conditions:

(1) For each $i, U_{i}: U_{i+2}: U_{i+1}$ or $U_{i+1}: U_{i+2}: U_{i}$, according as $i$ is odd or even;

(2) For each $i, j, G_{i, j}$ is contractible with respect to $U_{i}$ and $U_{i+1}$ and is of mesh less than $1 / i$;

(3) $G_{i, j} \gg G_{i-1, i-1}$ (if the latter is defined) and is a weak contraction of $G_{i, j+1}$;

(4) For each $i, U_{i}$ is a jumpless extension of $U_{i-2}$ with respect to $U_{i-1}, G_{i-1, i-1}$, and $G_{i, i}$;

(5) If $i \leqq j, i \leqq k$, then Ind $\left(U_{k}, U_{k-1}, G_{i, j}\right)$ is finite;

(6) If $g$ belongs to $G_{i, j}, g^{\prime}$ is the corresponding element of $G_{i, j+1}$ under the appropriate weak contraction, and $i^{\prime}>i$, then $g^{\prime}$ contains the closure of $g \cdot B\left(U_{i^{\prime}}\right)$; but no point of $B\left(U_{i}\right)$ is a limit point of $g^{\prime}-g$

(7) If $g$ belongs to $G_{i, j}, g^{\prime}$ belongs to $G_{i^{\prime}, j^{\prime}}\left(i^{\prime}>i\right)$, and $\bar{g} \cdot \bar{g}^{\prime}$ is not empty, then $T^{-1}(g)$ contains $\bar{g}^{\prime}$, where $T$ is the appropriate weak contraction; and

(8) Subject to all the above conditions, given $U_{1}, U_{2}, \cdots, U_{n}$

${ }_{14}$ To be exact, if $U_{2}: U_{1}$ and $g$ belongs to $G$, then Ind $\left(U_{2}, U_{1}, g\right)$ is the number of components of $g \cdot\left(D-\bar{U}_{2}\right)$ that have a limit point in $B\left(U_{1}\right)$, and Ind $\left(U_{2}, U_{1}, G\right)$ is $\sum_{\theta \in G}$ Ind $\left(U_{2}, U_{1}, g\right)$. As in several of the definitions and lemmas given above, we have a sort of duality relation in which certain sets $U, V$ are replaced by the complements (in $D$ ) of their closures. 
and $G_{i, j}(i \leqq n, j \leqq n)$, the immediately following terms $U_{n+1}$ and $G_{i, n+1}(i \leqq n+1)$ are chosen so as to minimize the numbers Ind $\left(U_{n+1}, U_{n-1}, G_{i, n+1}\right)=f(i)$ in the order of increasing $i$; that is to say, of all possible succeeding terms which satisfy (1)-(7) and which can be continued to give a complete sequence satisfying (1)-(7), we restrict ourselves to those for which $f(1)$ is minimal; among the latter possibilities, we limit ourselves to those for which $f(2)$ is minimal, and so on.

Of course we must start the sequences so that $R: U_{1}: U_{2}: R^{\prime}$. Let $G_{i}$, for each $i$, be the collection obtained by adding together the ascending sequences of corresponding elements of the collections $G_{i, j}$. Let $R^{\prime \prime}$ be the sum of the sets $U_{2 i-1}$. Then $B\left(R^{\prime \prime}\right)$ is the common part of the sets $G_{i}^{*}$.

One reason for using the triangular sequence $G_{i, j}$ rather than a simple sequence is that in order to obtain finite indices satisfying (5), we may need to enlarge our coverings slightly. On the other hand, in passing from $U_{i}$ to $U_{i+1}$, we do not need to enlarge the coverings in the neighborhood of $B\left(U_{i}\right)$, so that (6) can be satisfied. Note that (6) implies that (9) Ind $\left(U_{i+2 k}, U_{i}, G_{i, i+k^{\prime}}\right)$ is a nonincreasing function of $k$ and $k^{\prime}$ simultaneously. Note also that (4) and (7) imply that (10) for $k, k^{\prime}>0, U_{i+2 k}$ is a jumpless extension of $U_{i-2}$ with respect to $U_{i-1}$ and $G_{i, i+k}$.

It is clear that if there are sequences satisfying conditions (1)-(7), then there are sequences which also satisfy (8). To show that such sequences exist, we shall make use of an auxiliary triangular sequence $H_{i, j}$ which is to satisfy conditions (1)-(6) and also:

( $\left.7^{\prime}\right)$ If $h$ belongs to $H_{i, j}, Z$ is the sum of two intersecting closures of elements of $H_{i^{\prime}, j^{\prime}}\left(i^{\prime}>i\right)$ and $\bar{h}$ intersects $\bar{Z}$, then $T^{-1}(h)$ contains $\bar{Z}$, where $T$ is the weak contraction throwing $H_{i, j}$ into $H_{i, j+1}$.

We shall assume that $U_{i}$ and $H_{i, j}$ have been defined for $i \leqq j<n$ and that $G_{i, j}$ has been defined for $i \leqq j \leqq n-2$, and show that they may be extended a step further. In the transitivity step we shall assume that $n$ is odd, the corresponding argument when $n$ is even being entirely analogous.

We first define all the immediately succeeding terms of the $H$ - and $G$-sequences except for $H_{n, n}$ and $G_{n-1, n-1}$, so as to satisfy (1)-(7) and $\left(7^{\prime}\right)$. Given these, let $\epsilon$ be a positive number such that any set $H_{n, n}$ with mesh less than $\epsilon$ will satisfy $\left(7^{\prime}\right)$. By Lemma 2 , let $U_{n-1}^{\prime}$ be a set and let $H_{n-1, n-1}^{\prime}$ be a collection satisfying all the hypotheses for $H_{n-1, n-1}$ such that $U_{n-1}^{\prime}$ is a jumpless extension of $U_{n-1}$ with respect to $U_{n-2}, H_{n-1, n-1}^{\prime}$, and $H_{n-1, n-1}^{\prime}$, and such that $U_{n-1}^{\prime}$ is $\epsilon$-approachable from $U_{n-2}$ through $H_{n-1, n-1}^{\prime}$. Let $H_{n, n}$ be an admissible 
covering of $B\left(U_{n-1}^{\prime}\right)$, with the required finite indices, and let $U_{n}$ be an open set such that the mesh of $H_{n, n}$ is less than $\epsilon$, and such that $H_{n, n}$ is contractible with respect to $U_{n-1}^{\prime}$ and $U_{n}$. Let $G_{n-1, n-1}$ be the set obtained by replacing each element $h$ of $H_{n-1, n-1}$ by the sum $g$ of $h$ and all elements of $H_{n, n}$ that intersect $h$. By (10), $U_{n-1}^{\prime}$ is a jumpless extension of $U_{n-3}$ with respect to $U_{n-2}, G_{n-2}$, and $H_{n, n}$. We are now in the same position as before, with $n$ replaced by $n+1$.

LemMa 4. Let $p$ be a point of $g \cdot B\left(R^{\prime \prime}\right)$, where $g$ belongs to some $G_{i}$. Then there does not exist a sequence $C_{1}, C_{2}, \cdots$ of components of $R^{\prime \prime} \cdot g$ and a sequence $p_{1}, p_{2}, \cdots$ of points such that for each $j, p_{j}$ belongs to $C_{j}$, and such that $p$ is the sequential limit point of the sequence $p_{1}, p_{2}, \cdots$.

Proof. Since $R^{\prime \prime} \cdot g$ is locally connected, it contains no point of the limiting set of the $C$-sequence. Let $n$ be a natural number such that if $g^{\prime}$ and $g^{\prime \prime}$ belong to $G_{n}$ and have a point in common and $p \in g^{\prime}$, then $g^{\prime \prime} \subset g$. Let $P$ be the sum of all such elements $g^{\prime}$ of $G_{n}$. For each $2 i+1$ greater than $n, B\left(U_{2 i+1}\right)$ intersects $P$, and $P$ is the sum of two mutually separated sets $H$ and $K, H$ containing $P \cdot B\left(U_{2 i+1}\right)$ and $K$ intersecting a term of the $C$-sequence. This contradicts condition (10) for the $U$-sequence.

LEMmA 5. Let $p$ and $g$ be as in Lemma 4. Then there do not exist two components $C$ and $C^{\prime}$ of $R^{\prime \prime} \cdot g$, each of which has $p$ as a limit point.

Proof of Lemma. Assume that the lemma is false, let $n$ be an even integer, and let $g_{n}$ be an element of $G_{n, n}$ containing $p$. Let $k$ be the least odd integer for which $g_{n}$ contains a point of $C \cdot B\left(U_{k}\right)$ and a point of $C^{\prime} \cdot B\left(U_{k}\right)$. Then $C$ and $C^{\prime}$ contribute a count of at least two to Ind $\left(U_{k+2}, U_{k}, G_{n, k+2}\right)$. We shall show that if the lemma is false, then $U_{k+2}$ did not satisfy condition (8).

Let $G_{n, k+1}^{\prime}$ be $G_{n, k+2}$. Let $G_{k+1, k+1}^{\prime}$ be a certain $G_{m, m}, m$ being an odd number, sufficiently large so that condition (7) will be satisfied. Let $P$ be an open set containing $p$ and lying in an element of $G_{m, m}$ such that $P \cdot D$ is connected, and such that $\bar{P}$ lies in $U_{m+3}$. Replace $U_{k+2}$ by some odd-numbered element of the $U$-sequence which intersects $P$. By this process Ind $\left(U_{k+2}, U_{k}, G_{n, k+2}\right)$ has been reduced. But given any $\epsilon$, we may choose $P$ and $U_{k+2}^{\prime}$ so as to make $U_{k+2}^{\prime} \epsilon$-approachable from $U_{k+1}$ through $G_{m, m}$, so that the sequence can be continued satisfying conditions (1)-(7). Ind $\left(U_{k+2}, U_{k}, G_{n, k+2}\right)$ was therefore not minimal, which contradicts condition (8), and the lemma is proved. The theorem now follows immediately.

Definition 1. Let $S$ be a Peano space and let $G$ be a finite collection 
of mutually exclusive connected open sets such that $\bar{G}^{*}$ is $S$. Then $G$ is a connected grating decomposition of $S$. If the elements of $G$ are uniformly locally connected, then $G$ is a grille decomposition of $S$. Two elements of $G$ are adjacent if they have a boundary point in common. If $G_{1}, G_{2}, \ldots$ is a sequence of connected grating (or grille) decompositions of $S$ such that each term of the sequence after the first is a refinement of its predecessors, and such that for each positive $\epsilon$, only a finite number of terms of the sequence have mesh greater than $\epsilon$, then the sequence $G_{1}, G_{2}, \ldots$ is a complete sequence of connected grating (or grille) decompositions of $S$.

Theorem 2. Every Peano space has a complete sequence of grille decompositions.

Proof. Let $H$ and $H^{\prime}$ be finite coverings of $S$ by open sets such that $H^{\prime} \gg H$. Let $h$ and $h^{\prime}$ be elements of $H$ and $H^{\prime}$ respectively such that $\bar{h}^{\prime} \subset h$. We now have the hypothesis of Theorem 1 satisfied with $D=S$. Let $g_{1}$ be the set $R^{\prime \prime}$ of the conclusion of Theorem 1 such that $h^{\prime}: g_{1}: h$. Now let the $D$ of Theorem 1 be $S-\bar{g}_{1}$; we choose another element of $H^{\prime}$, and an element of $H$ containing its closure; and we proceed as before to obtain a set $g_{2}$ which contains $\bar{h}^{\prime \prime} \cdot D$. At each stage, we let $D$ be the complement of the closure of the sum of the sets $g_{i}$ previously obtained. Finally we let $G_{1}$ be the set of all components of the sets $g_{i}$. To obtain $G_{2}$, we work with the elements $g$ of $G_{1}$ one at a time, starting the process for each $g$ by letting $D=g$.

Definition 2. Let $G_{1}, G_{2}, \cdots$ be a complete sequence of connected grating decompositions of the Peano space $S$; and let $g \in G_{i}$. Then the border Bord $(g)$ of $g$ is the set of all elements $g^{\prime}$ of $G_{i+1}$ that lie in $g$ and have a boundary point in common with $\beta(g)$. The core Core $(g)$ of $g$ is the set of all elements of $G_{i+1}$ that lie in $g$ but do not belong to Bord $(g)$.

Definition 3. A chain is a finite collection $C$ of mutually exclusive open sets $c_{1}, c_{2}, \cdots, c_{k}$, such that the sets $c_{i}$ and $c_{j}$ have a boundary point in common if and only if $i$ and $j$ are identical or consecutive integers. The end-links of the chain are $c_{1}$ and $c_{k}$. If $x$ and $y$ are points of $c_{1}$ and $c_{k}$ respectively (or of $\bar{c}_{1}$ and $\bar{c}_{k}$ respectively), then $C$ is a chain from $x$ to $y$ (or a chain spanning $x$ and $y$ ). If $H$ and $K$ are sets intersecting $\bar{c}_{1}$ and $\bar{c}_{k}$ respectively, then $C$ spans $H$ and $K$.

Definition 4. A complete sequence of connected grating decompositions is core-wise connected if for each $g$ of each $G_{i}$ and for each pair $g^{\prime}, g^{\prime \prime}$ of elements of $G_{i+1}$ lying in $g$, there is a chain of elements of $G_{i+1}$, having $g^{\prime}$ and $g^{\prime \prime}$ as its end-links, such that $C-g^{\prime}-g^{\prime \prime}$ lies in Core $(g)$. 
Theorem 3. Every Peano space has a core-wise connected complete sequence of grating decompositions.

Proof. Let $G_{1}, G_{2}, \cdots$ be a complete sequence of connected grating decompositions of the space $S$. Let $g$ be an element of $G_{1}$. By deleting a finite number of terms of the $G$-sequence, if necessary, we may arrange for Core $(g)$ not to be empty. Let $x$ be a point belonging to an element of Core $(g)$. For each $h$ of $G_{2}$ lying in $g$, let $x_{h}$ be a point of $h$. Let $A$ be a collection of arcs from $x$ to the points $x_{h}$, not intersecting $\beta(g)$. Let $n$ be an integer such that if $g_{n}$ belongs to $G_{n}$, then $\bar{g}_{n}$ does not intersect both $A^{*}$ and $\beta(g)$. Let $Z$ be a collection of chains of elements of $G_{n}$, no links of which have boundary points in common with $\beta(g)$, and which contain $x$ and the points $x_{h}$ in the closures of their end-links. (Note that $x$ and the points $x_{h}$ do not necessarily belong to $G_{n}^{*}$.) For each $h$ of Bord $(g)$, let $G_{h}$ be the set of all components of $h-\bar{Z}^{* *}$ that have a boundary point in $\beta(g)$. Since each $h$ is connected, each element of $G_{h}$ will have a boundary point in $\bar{Z}^{* *}$. Let $G_{h}^{\prime}$ be the set of all components of $h-\bar{G}_{h}^{*}$. Now let $G$ be the sum of Core $(g)$, the sets $G_{h}$, and the sets $G_{h}^{\prime}$.

Let $G_{1}^{\prime}$ be $G_{1}$ and let $G_{2}^{\prime}$ be $G . G_{1}^{\prime}$ is clearly corewise connected and the same process may be repeated to give a complete sequence.

\section{Theorem 4. Every Peano space can be given a convex metric.}

Proof. Let $G_{1}, G_{2}, \cdots$ be a complete sequence of core-wise connected grating decompositions of the space $S$. We shall define a certain subsequence $G_{1}^{\prime}, G_{2}^{\prime}, \cdots$ of the $G$-sequence. For each $g$ of each $G_{i}$, we shall define a real number $\delta(g)$. For each two points $x, y$ of $S$, we shall let $d_{t}^{\prime}(x, y)$ be the least real number $d$ for which there is a chain $C: c_{1}, c_{2}, \cdots, c_{k}$ of elements of $G_{i}^{\prime}$ such that $x$ and $y$ belong to $\bar{c}_{1}$ and $\bar{c}_{k}$ respectively, and such that the length $l(c)=\sum \delta\left(c_{j}\right)$ of the chain $C$ is equal to $d$.

For each $g$ of $G_{1}^{\prime}=G_{1}$, let $\delta(g)=1$.

Now suppose that $G_{1}^{\prime}, \cdots, G_{i}^{\prime}$ have been defined as a subsequence of the $G$-sequence and the $\delta$ function has been defined for each of their elements. Let $G_{i+1}^{\prime}$ be a term of the $G$-sequence following $G_{i}^{\prime}$ such that no element of $G_{i}^{\prime}$ has an empty core and satisfying a second condition which we shall state presently. Let $p$ be the number of elements of $G_{i+1}^{\prime}$ and let $\eta$ be $2^{-i-2} / p$. For each $g$ of $G_{i}^{\prime}$ and each $b$ of Bord $(g)$, let $\delta(b)$ be $(1 / 2)(\delta(g)-\eta)$. Let $q$ be the number of elements of Core $(g)$, and for each $c$ of Core $(g)$, let $\delta(c)$ be $\eta / q$. The second condition imposed on $G_{i+1}^{\prime}$ is that with the above definition of $\delta$, any chain of elements of $G_{i+1}^{\prime}$ which consists entirely of elements of the borders of elements of $G_{i}^{\prime}$ and spans two non-adjacent elements 
of $G_{i}^{\prime}$ shall have length greater than that of any chain of $G_{i}^{\prime}$. Since $\delta$ is an increasing function of $p$ on the borders of elements of $G_{i}^{\prime}$, this condition may be satisfied merely by going out far enough in the $G$-sequence.

Let $m$ be the least of $\eta$ and all numbers $\delta(b)$, for each $c$ of each Core $(g)$, let $\delta(c)=m / p$.

Lemma 1. Let $x$ and $y$ be points of $S$ and let $i$ be an integer such that no chain of elements of $G_{i}^{\prime}$ of less than five links spans $x$ and $y$. For each $j$ greater than $i$, let $d_{j}^{\prime \prime}(x, y)$ be the length of the shortest chain of elements of $G_{j}^{\prime}$ which spans the sum of (1) the set of all points $x$ for which there is a two-link chain of elements of $G_{i}^{\prime}$ spanning $x$ and $x^{\prime}$ and (2) the set of all points $y^{\prime}$ for which there is such a chain spanning $y$ and $y^{\prime}$. Then $D_{j+1}^{\prime \prime}(x, y) \geqq\left(1-2^{-j}\right) d_{j}^{\prime \prime}(x, y)$.

Proof of Lemma. Let $C^{\prime}$ be a chain of elements of $G_{j+1}^{\prime}$ spanning (1) and (2) and let $C$ be the set of all elements of $G_{j}^{\prime}$ that contain one or more elements of $C^{\prime}$, with the ordering naturally induced by that of $C^{\prime}$. If we can show that each $c$ of $C$ contains two links of $C^{\prime}$ that belong to Bord $(c)$, the lemma will be proved. Suppose that there is a $c$ of $C$ which does not have this property and let $C_{c}$ be a subcollection of $C$ which is maximal with respect to being a segment of consecutive elements of $C$ none of which have the property. Then every element of $C_{c}$ is adjacent to every other element of $C_{c}$ and has a boundary point in common with (1) or (2).$^{15}$ Suppose, without loss of generality, that each element of $C_{c}$ has a boundary point in common with (1). It follows that the first element $c_{1}$ of $C$ that follows all elements of $C_{c}$ also has a boundary point in common with (1); and this $c_{1}$ contains two links of $C^{\prime}$ that lie in Bord $\left(c_{1}\right)$. But there is an element of Bord $\left(c_{1}\right)$ that has a boundary point in common with (1); $C^{\prime}$ may therefore be modified in an obvious manner so as to reduce its length, which contradicts our hypothesis.

LEMMA 2. If $x$ and $y$ are different points of $S$, then $d(x, y)=\lim _{i \rightarrow \infty}$ $d_{i}^{\prime}(x, y)$ exists and is positive.

Proof of Lemma. Since, for fixed $x, y, d_{i}^{\prime}$ is a diminishing function of $i$, we need only show that its limit is not zero. It is obvious that $d_{j}^{\prime}$ is not less than any $d_{j}^{\prime \prime}$ of the type defined in Lemma 1; our result therefore follows directly from the convergence of the infinite product $\Pi\left(1-2^{-i}\right)$.

Lemma 3. If $x$ is the sequential limit of the sequence $x_{1}, x_{2}, \ldots$

${ }^{16}$ This can be shown by an argument like the one immediately following. 
of points of $S$, then $\lim _{i \rightarrow \infty} d\left(x, x_{i}\right)=0$, and conversely.

Lemma 4. If $x, y$, and $z$ belong to $S$, then $d(x, y)+d(y, z) \geqq d(x, z)$.

LEMma 5. If $x$ and $z$ belong to $S$, then there is a point $y$ such that $d(x, y)=d(y, z)=(1 / 2) d(x, z)$.

Proof of Lemma. By the construction of the distance function, it is clear that for each $i$ there is a point $y_{i}$ for which the required equalities hold with an error of less than $1 / i$. Any point $y$ which appears infinitely often in the sequence, or which is a limit point of $\sum y_{i}$, satisfies the conclusion of the lemma.

It follows that $d$ is a convex metric.

UNIVERSITY OF MichigAN 